\title{
Cardiovascular complications of SARS-CoV-2 infection (COVID-19): a systematic review and meta-analysis
}

Yu-Hao Zhao ${ }^{1, \dagger}$, Lei Zhao ${ }^{1, \dagger}$, Xin-Chun Yang $^{1, *}$, Pan Wang ${ }^{1, *}$

${ }^{1}$ Heart Center \& Beijing Key Laboratory of Hypertension, Beijing Chaoyang Hospital, Capital Medical University, 100020 Beijing, P. R. China

"Correspondence: diudiupan@163.com (Pan Wang); yangxinchun6229@163.com (Xin-Chun Yang)

${ }^{\dagger}$ These authors contributed equally.

DOI:10.31083/j.rcm.2021.01.238

This is an open access article under the CC BY 4.0 license (https://creativecommons.org/licenses/by/4.0/).

Submitted: 05 November 2020 Revised: 04 January 2021 Accepted: 05 January 2021 Published: 30 March 2021

Coronavirus Disease 2019 (COVID-19) originated in Wuhan, China in December 2019 and rapidly spread worldwide. Herein, we conducted a systematic review and meta-analysis to find the association between COVID-19 and cardiovascular complications. We conducted a systematic literature search of the PubMed and Embase databases from 01 December 2019 to 30 November 2020. We then statistically analyzed the incidence of cardiovascular complications in COVID-19 patients. We included 3044 confirmed COVID-19 cases from 12 studies. The most common cardiovascular complications in COVID-19 patients were myocardial injury $(21.2 \%, 95 \% \mathrm{Cl} 12.3-30.0 \%)$ and arrhythmia $(15.3 \%, 95 \% \mathrm{Cl} 8.4-22.3 \%)$, followed by heart failure $(14.4 \%$, $95 \% \mathrm{Cl} 5.7-23.1 \%)$ and acute coronary syndrome (1.0\%, $95 \% \mathrm{Cl} 0.5-$ $1.5 \%)$. The pooled incidence of heart failure, arrhythmia and myocardial injury in non-survivors were $47.8 \%(95 \% \mathrm{Cl} 41.4-54.2 \%), 40.3 \%$ (95\% $\mathrm{Cl} 1.6-78.9 \%)$ and $61.7 \%(95 \% \mathrm{Cl} 46.8-76.6 \%)$, respectively. Also, the data separately showed significantly higher incidence of heart failure and cardiac injury in non-survivors (relative risks $=5.13,95 \%$ $\mathrm{Cl} 2.46-10.7, \mathrm{Z}=4.36, P=0.017$ ) and (relative risks $=6.91,95 \% \mathrm{Cl} 3.19$ 14.95, $Z=4.91, P=0.009$ ). Myocardial injury and arrhythmia were the most common complications in COVID-19 patients. Myocardial injury and heart failure were more common in patients who died, regardless of a history of cardiovascular disease. The incidence of heart failure and myocardial injury were higher in non-survivors compared to the survivors. Accordingly, in addition to basic support, cardiac reactions of patients with confirmed COVID-19 with or without underlying cardiovascular diseases should be closely monitored.

\section{Keywords}

COVID-19; Cardiovascular complications; Heart failure; Myocardial injury; Acute coronary syndrome; Arrhythmia

\section{Introduction}

Since the first case was reported in December 2019 in Wuhan, China, the novel coronavirus disease (COVID-19) has spread and become a global pandemic. Up to Nov 30, 2020, a total of $62,195,274$ confirmed cases of COVID-19 have been reported globally, with 93,465 cases in China and $62,101,809$ cases outside of China, and 1,453,355 reported deaths [1]. The pathogen for this disease has been identified and subsequently named as severe acute respiratory syndrome coronavirus 2 (SARS-CoV-2) by the World Health Organization (WHO).

SARS-CoV-2 resembles SARS-CoV clinically and epi- demiologically [2], but it appears to be more transmissible and virulent than SARS and MERS-associated coronaviruses. Although respiratory symptoms are one of the common initial clinical presentation (e.g., dry cough, dyspnea) of COVID-19, emerging reports have found severe morbidity and mortality associated with cardiovascular comorbidities [3].

Some possible mechanisms by which SARS-CoV-2 causes cardiovascular disease have been proposed. The direct myocardial effects of SARS-CoV-2 may be one of the causes of adverse cardiac outcomes in COVID-19 patients [4]. SARSCoV-2 is thought to enter into the host cell through the human angiotensin converting enzyme-2 (ACE-2) receptor, a surface molecule that is localized in arterial smooth muscle, respiratory tract epithelium, arterial and venous endothelial cells, intestinal epithelial cells, and immune cells, after the priming of spike protein by host cell proteases [5, 6]. The combination of SARS-CoV-2 and ACE-2 can alter the ACE2 signaling pathway and directly lead to myocardial injury.

Inflammatory cytokine storm during COVID-19 probably accelerates the disease progression. A recent case report indicated increased Th17 and high cytotoxicity of CD8 T cells in COVID-19 [7]. It has been speculated that inflammatory cytokines released due to dysfunctional immune response in viral infection partly contribute to cardiac dysfunction, which in turn leads to systemic inflammation that may trigger rupture or erosion of coronary plaques and arrhythmia [7].

Since alveolar cells have high expression of ACE-2, the potentially increased transmissibility and severe lung injury could be the main clinical characteristics. Cardiologists are also concerned about whether new coronavirus infections can affect the cardiovascular system. A recent study reported that COVID-19 can cause heart injury, even in patients without underlying heart problems [4].

To date, many critically ill COVID-19 patients remain hospitalized. Whether cardiovascular diseases are common in patients infected with COVID-19 remains uncertain. Given that cardiovascular complications can predict the disease progression, we conducted a systematic literature search for evidence of cardiovascular diseases among COVID-19 patients. 


\section{Materials and methods \\ 2.1 Search strategy and selection criteria}

We searched the electronic databases of PubMed and Embase from Dec 1, 2019 to Nov 30, 2020 using the keywords ("2019 novel coronavirus" OR "2019-nCoV" OR "SARSCoV-2” OR “COVID-19”) AND (“cardiovascular disease” OR "cardiac complications" OR "heart failure" OR "arrhythmia" OR "acute coronary syndrome" OR "myocardial infarction" OR "atrial fibrillation" OR "myocarditis" OR "hypertension"). We applied no language restrictions and reviewed the references of included articles to extend the search.

A total of 12 articles were included. The inclusion criteria were as follows: (1) patients were confirmed to have COVID-19; (2) at least one outcome of new cardiovascular diseases was reported; (3) study sample was larger than 10 patients. We excluded duplicate reports, commentary, case reports and studies with insufficient information, as well as studies written in Chinese (to avoid data duplication).

\subsection{Study selection, data extraction and synthesis}

Two investigators (ZYH and ZL) independently screened all titles and abstracts identified in our literature search. All available information, including baseline characteristics and cardiovascular complications, was recorded using a Microsoft Excel database. Any disagreement was resolved by other investigators (YXC and WP).

\subsection{Statistical analysis}

All analyses were performed using Microsoft Excel and Stata software version 14.0. The results of the included studies were analyzed by the random-effects model or the fixedeffects model in cases of significant heterogeneity between studies. The $\mathrm{I}^{2}$ statistic was used to quantify the heterogeneity across studies. If the results were homogeneous $\left(\mathrm{I}^{2}\right.$ $<50 \%$ ), fixed-effects models were utilized, while if the results were heterogeneous ( $\mathrm{I}^{2} \geq 50 \%$ ), random-effects models were used. Summary relative risks (RRs) with 95\% CIs were estimated for the association between survivors and non-survivors. Publication bias was assessed by a funnel plot and Begg's test. A $P$-value $<0.05$ was considered as statistical significance.

\section{Results \\ 3.1 Research selection and quality assessment}

The initial search produced 5775 potentially relevant articles. After deleting duplicate records and primary screening, 57 articles were included in the meta-analysis through fulltext evaluation. Of these, 45 were excluded because 43 articles did not report one new cardiovascular disease complication of COVID-19 and two were review articles. Finally, a total of 12 studies with 3044 patients were included in the metaanalysis [4, 8-18] (Fig. 1). The sample sizes of the selected studies varied from 41 to 1000 patients. The demographic data and characteristics of the included studies are shown in Tables 1,2.

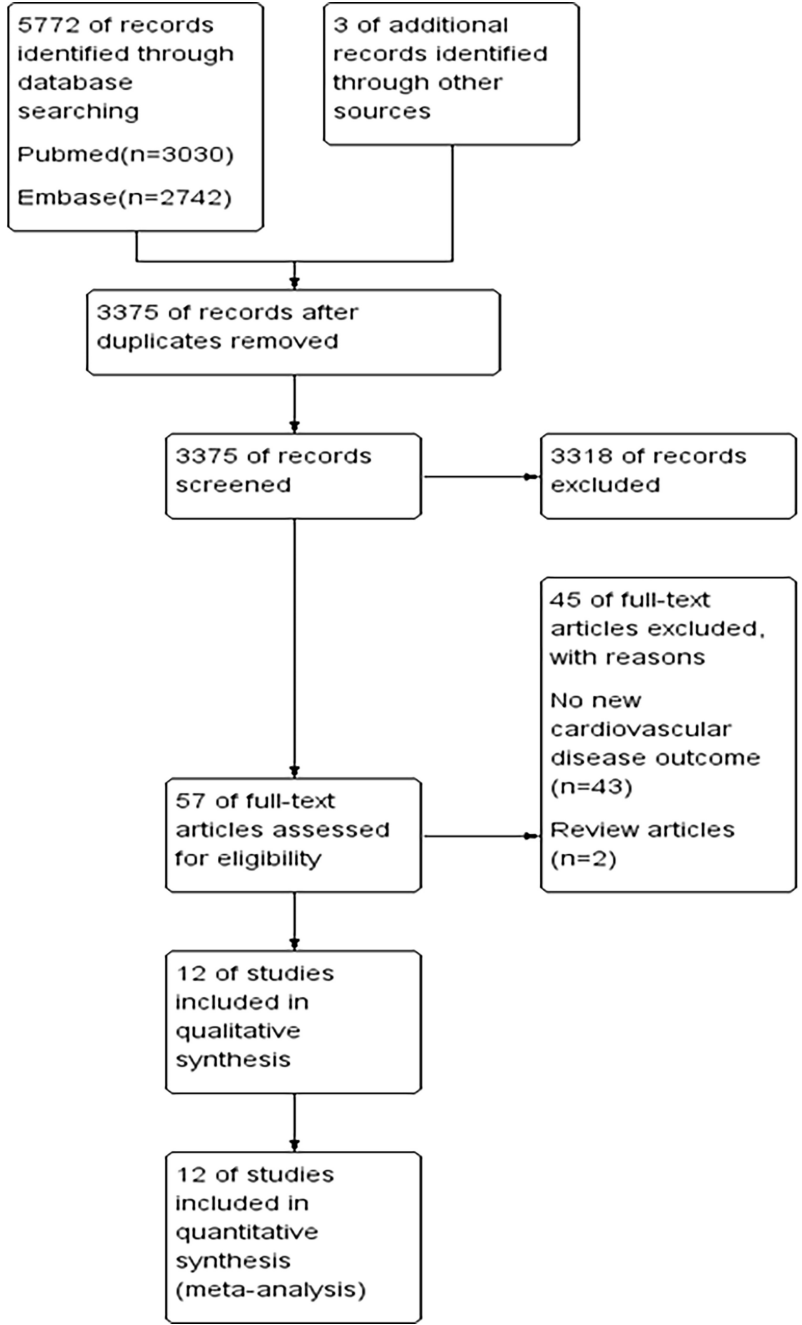

Fig. 1. Flow diagram showing the process of the systematic search. The number of studies is the bottom of the flowchart represents that of the selected studies that were considered eligible for inclusion in this metaanalysis.

\subsection{Primary outcomes}

Meta-analysis of the identified studies showed that the most common cardiovascular complications in COVID-19 patients were myocardial injury $(21.2 \%$, 95\% CI 12.3-30.0\%) and arrhythmia (15.3\%, 95\% CI 8.4-22.3\%), followed by heart failure $(14.4 \%$, 95\% CI $5.7-23,1 \%)$ and acute coronary syndrome (1.0\%, 95\% CI 0.5-1.5\%). There was a significant heterogeneity in the estimates of complications among the identified studies, with an $\mathrm{I}^{2}$ of 0-96.7\% (Fig. 2).

Four studies reported non-survivors. The pooled incidences of heart failure, arrhythmia and myocardial injury in non-survivors were $47.8 \%$ (95\% CI 41.4-54.2\%), 40.3\% (95\% CI $1.6-78.9 \%$ ) and $61.7 \%$ (95\% CI 46.8-76.6\%), respectively (Fig. 3).

We then compared the difference of the prevalence of heart failure and myocardial injury between survivors and non-survivors. The result indicated a statistically higher incidence of heart failure in non-survivors compared to the sur- 
Table 1. Main Characteristics of included studies in the meta-analysis.

\begin{tabular}{|c|c|c|c|c|c|c|c|c|}
\hline \multirow{2}{*}{ References } & \multirow{2}{*}{ Date } & \multirow{2}{*}{ Number of patients } & \multirow{2}{*}{ Sex (male) } & \multirow{2}{*}{ Age } & \multicolumn{4}{|c|}{ Complications } \\
\hline & & & & & HF (\%) & ACS (\%) & Arrhythmia (\%) & Myocardial injury (\%) \\
\hline Wang. D.W. et al. [16] & 2020.01.01-2020.01.28 & 138 & 63 & $56(42-68)$ & - & - & 16.7 & 7.2 \\
\hline Du et al. [11] & 2020.01.09-2020.02.05 & 85 & 62 & 65.8 & - & 4.9 & 60 & 44.7 \\
\hline Huang et al. [4] & $2019.12 .16-2020.01 .02$ & 41 & 30 & $49(41-58)$ & - & - & - & 12 \\
\hline Guo et al. [12] & $2020.01 .23-2020.02 .23$ & 187 & 91 & 58.5 & - & - & 7 & - \\
\hline Wan et al. & $2020.01 .23-2020.02 .08$ & 135 & 72 & $47(36-55)$ & - & - & - & 7.4 \\
\hline Wang L. et al. [15] & 2020.01.01-2020.02.06 & 339 & 165 & $69(65-76)$ & 17.4 & - & 10.4 & 21 \\
\hline Chen et al. [10] & $2020.01 .13-2020.02 .12$ & 274 & 171 & $62(44-70)$ & $25(44 / 177)$ & - & - & $44(90 / 203)$ \\
\hline Zhou et al. [17] & As of 2020.01 .31 & 191 & 119 & $56(46-67)$ & 23 & - & - & 17 \\
\hline Shi et al. [14] & $2020.01 .01-2020.03 .01$ & 161 & 104 & 59.38 & 5.59 & 1.24 & 1.86 & - \\
\hline Lodigiani et al. [13] & $2020.02 .12-2020.04 .10$ & 388 & 426 & $66(55-75)$ & - & 1 & - & - \\
\hline Argenziano et al. [8] & 2020.03.01-2020.04.05 & 1000 & 596 & $63(50-75)$ & $2.8(24 / 850)$ & $0.9(8 / 850)$ & $9.3(79 / 850)$ & - \\
\hline Buckner et al. [9] & 2020.03.02-2020.03.26 & 105 & 53 & $69(23-97)$ & - & - & - & $19(13 / 67)$ \\
\hline
\end{tabular}

HF: heart failure; ACS: acute coronary syndrome.

Table 2. Three studies compared survivor patients and non-survivor patients.

\begin{tabular}{|c|c|c|c|c|c|c|c|}
\hline \multirow{2}{*}{ References } & \multirow{2}{*}{ Number of patients } & \multirow{2}{*}{ Sex (male) } & \multirow{2}{*}{ Age } & \multicolumn{4}{|c|}{ Complications } \\
\hline & & & & $\mathrm{HF}(\%)$ & $\operatorname{ACS}(\%)$ & Arrhythmia (\%) & Myocardial injury (\%) \\
\hline \multirow{2}{*}{ Wang L. et al. [15] } & $274(S)$ & 126 & $68(64-74)$ & 12.1 & - & 8.1 & 11.4 \\
\hline & 65 (D) & 39 & $76(70-83)$ & 42.4 & - & 20.6 & 65 \\
\hline \multirow{2}{*}{ Chen et al. [10] } & $161(\mathrm{~S})$ & 88 & $51(37-66)$ & $3(3 / 94)$ & - & - & $17(18 / 109)$ \\
\hline & $113(\mathrm{D})$ & 83 & $68(62-77)$ & $49(41 / 83)$ & - & - & $77(72 / 94)$ \\
\hline \multirow{2}{*}{ Zhou et al. [17] } & $137(\mathrm{~S})$ & 81 & $52(45-58)$ & 12 & - & - & 1 \\
\hline & 54 (D) & 38 & $69(63-76)$ & 52 & - & - & 59 \\
\hline
\end{tabular}

HF: heart failure; ACS: acute coronary syndrome; S: survivor; D: death.

vivors $(\mathrm{RR}=5.13,95 \% \mathrm{CI} 2.46-10.7, \mathrm{Z}=4.36, P=0.017)$. The heterogeneity test showed $\mathrm{I}^{2}=75.3 \%$. Thus, the randomeffects model was used for the meta-analysis. Similarly, the data showed a significantly higher incidence of myocardial injury in non-survivors $(\mathrm{RR}=6.9195 \% \mathrm{CI} 3.19-14.95, \mathrm{Z}=$ $4.91, P=0.009)$. The heterogeneity test showed $\mathrm{I}^{2}=78.7 \%$ and the random-effects model was used. A funnel plot was drawn to detect publication bias (Fig. 4). Begg's test of heart failure and myocardial injury were $P=0.296>0.1$ and $P=1.0$ $>0.1$, respectively, which indicated that there was no publication bias.

\section{Discussion}

Although COVID-19 is a respiratory disease and respiratory symptoms are the initial clinical presentation (e.g., dry cough, dyspnea), accumulating evidence have suggested that COVID-19 is closely associated with cardiovascular complications. Wang et al. found that about $16.7 \%$ and $7.2 \%$ of COVID-19 patients developed arrhythmias and myocardial injury [18], respectively, while Du et al. and Chen et al. found that $4.9 \%$ and $25 \%$ of COVID-19 patients suffered from acute coronary syndrome and heart failure $[10,11]$. The higher incidences of cardiac arrhythmias, heart failure-related events and acute coronary syndromes during seasonal influenza outbreaks suggests that acute respiratory infections may lead to cardiovascular issues through proinflammatory effects, activation of coagulation pathways or endothelial cell dysfunction [19].

In this systematic review and meta-analysis, myocardial injury and arrhythmia were the most common complications in COVID-19 patients, and the incidences of heart failure and myocardial injury were about five-folds and seven-folds, respectively, which were higher in non-survivors compared to the survivors. Myocardial injury and heart failure were more common in non-survivors, regardless of a history of cardiovascular disease.

SARS-CoV-2 is an RNA virus, which is a member of the coronavirus family, similar to SARS-CoV [6]. Reported that the SARS-CoV RNA was detected in human heart samples at autopsy, indicating direct invasion of cardiomyocytes by the virus. They also found a significantly down-regulated expression of ACE-2, which may explain myocardial dysfunction and adverse cardiac outcomes in patients with SARS. TNF- $\alpha$ is a common inflammatory cytokine. The downregulation of ACE-2 impedes cardioprotective effects of angiotensin 1-7, provokes inflammatory cascade and promotes TNF- $\alpha$ production [6], which suggest that severe inflammatory response may be mediators of cardiomyocyte damage. It has been proposed that in patients with SARS, a strong interferon-mediated response may lead to myocardial dys- 


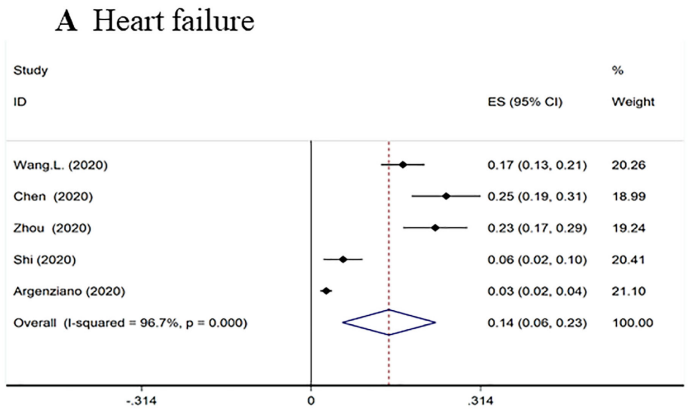

B Acute coronary syndrome
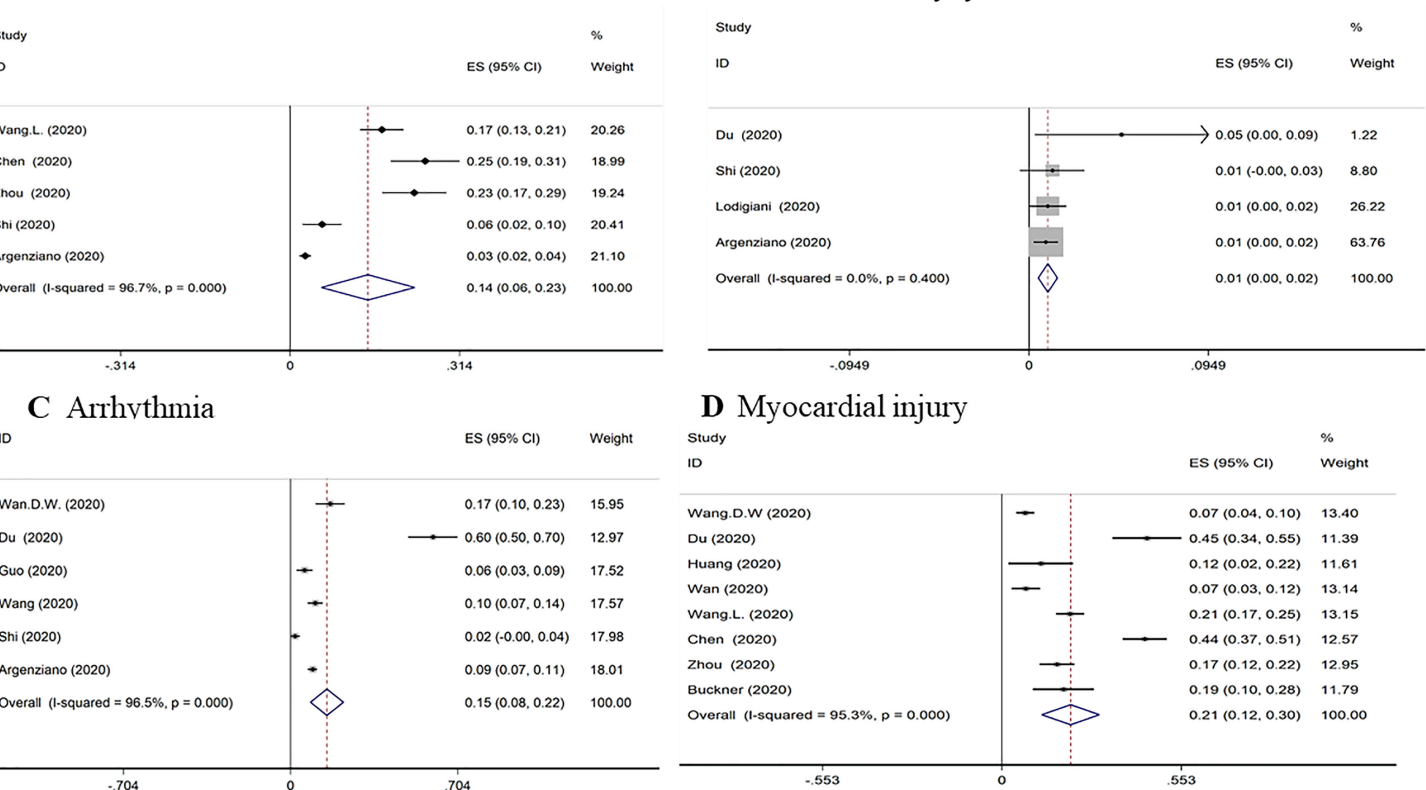

Fig. 2. Forest plots of incident cardiac complications after COVID-19. Each square shows effect estimate of individual studies with their 95\% CI. Size of squares is proportional to the weight of each study in the meta-analysis. In this plot, studies are shown in the order of publication date and first author's names.

function [20]. Gaaloul et al. reported that myocardial inflammation caused by viral infection leads to electrophysiological and structural remodeling or ion channel dysfunction, which is one of the mechanisms for arrhythmia [21].

The mechanisms of COVID-19 leading to cardiovascular complications are currently being investigated. First, the SARS-CoV subfamily is known to enter cells via ACE-2 receptors, which also causes direct damage to the myocardium [22], and the higher expression of ACE-2 has been postulated to enhance susceptibility to SARS-CoV-2 in patients with hypertension and cardiovascular disease [23]. Second, COVID19 causes severe acute systemic inflammatory responses and cytokine storms that lead to irreversible multi-organ damage. A recent case report indicated increased Th17 and high cytotoxicity of CD8 T cells in COVID-19 [7]. Also, systemic inflammation leads to increased coronary blood flow and higher shear stress, which precipitate plaque rupture and accelerate acute myocardial infarction. Clinical studies have identified that the risk of acute coronary syndrome significantly increase soon after the development of respiratory infections [24]. Hamadeh et al. also reported that a high rate of thrombotic complications and a very high mortality rate in patients presenting COVID-19 and ST-Segment elevation myocardial infarction [25]. Existing evidence suggests that viral infections may play a role in arrhythmia in COVID-19 patients [21]. Elsaid et al. reported that a patient who infected COVID-19 alone without hydroxychloroquine experienced long QT interval and polymorphic ventricular tachycardia [26]. Third, hypoxemia probably results in increased pulmonary arterial pressure and right ventricular afterload, while impairing myocardial oxygen delivery [27]. In Huang's study [4], 32\% of COVID-19 patients showed varying degrees of hypoxemia and required high-flow nasal cannula or higher-level oxygen support. Chen et al. also reported that up to $76 \%$ of patients received oxygen therapy [28]. Systemic infection caused by acute respiratory diseases and increased cardiometabolic demand caused by hypoxia impair myocardial oxygen demand-supply relationship and promote myocardial injury [29]. Lastly, several drugs currently used in the treatment of COVID-19 have deleterious effects on the cardiovascular system [23]. Heart failure, conduction defects and ventricular arrhythmias have been reported during azithromycin and remdesivir therapy [30, 31]. Chorin et al. [32] also found that in a significant proportion of patients, treatment of COVID-19 with hydroxychloroquine/azithromycin prolongs the QTc to an extreme degree and increase the risk for torsade de pointes.

While heart failure, myocardial injury, cardiac arrhythmias and acute coronary syndrome present different clinical characteristics, they also have shared pathophysiological basis and risk factors. Clinicians are currently focusing on the most common and fatal manifestations of COVID-19. However, cardiac complications are becoming more prevalent with the progress in the study of COVID-19. In summary, patients infected with SARS-CoV-2 may face the risk of cardiovascular complications, which influences the development and prognosis of disease. Briedis et al. have provided suggestions for management of acute coronary syndrome during the COVID-19 pandemic [33]. Moreover, viral infection-related heart damage should be closely monitored during the course of COVID-19 treatment. 
A Heart failure

Study

ID

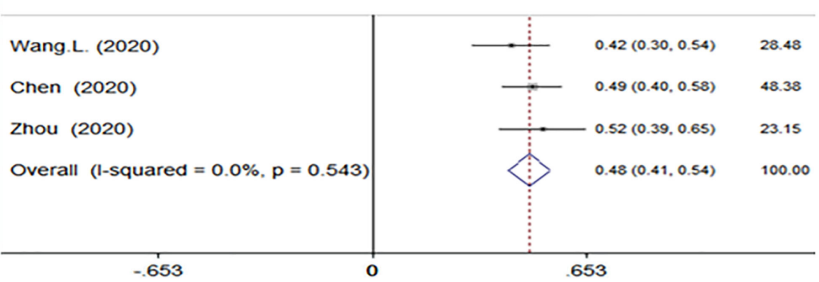

C Myocardial injury

\section{B Arrhythmia}

Study

ID

ES $(95 \% \mathrm{Cl}) \quad$ Weight

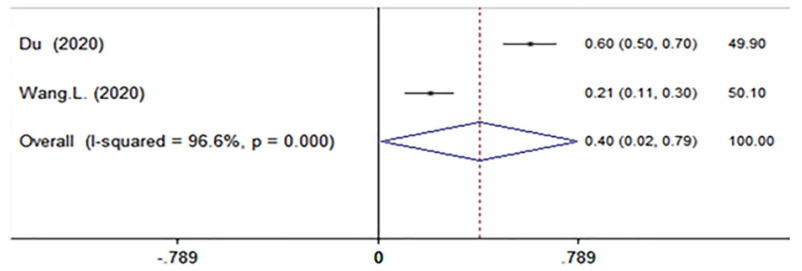

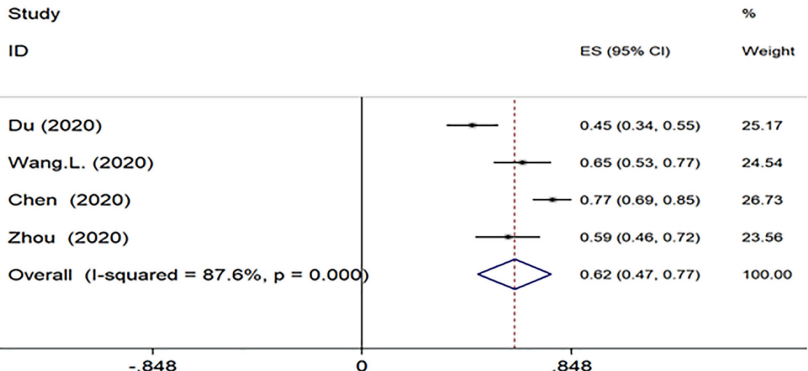

Fig. 3. Forest plots of incident cardiac complications after COVID-19 in non-survivors. Each square shows effect estimate of individual studies with their $95 \%$ CI. Size of squares is proportional to the weight of each study in the meta-analysis. In this plot, studies are shown in the order of publication date and first author's names.

\section{A Heart failure}

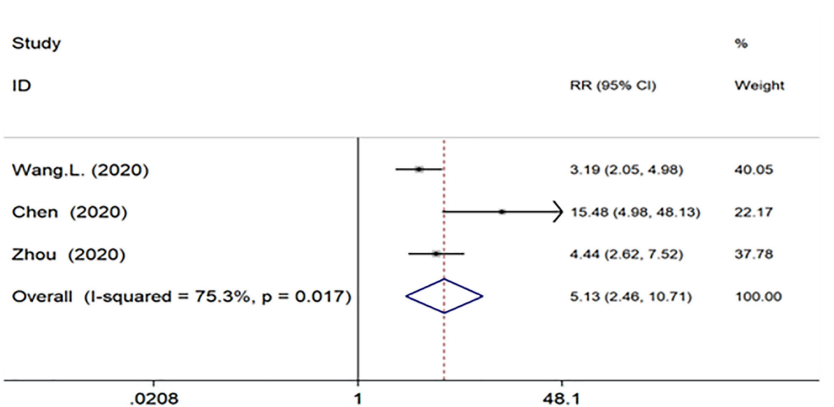

B Myocardial injury

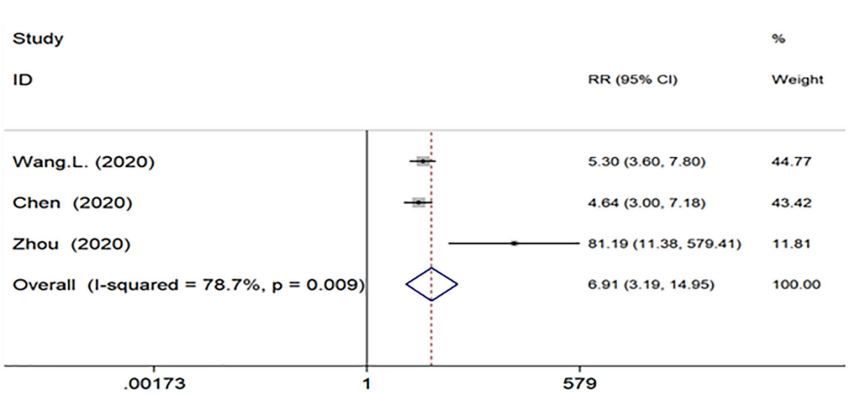

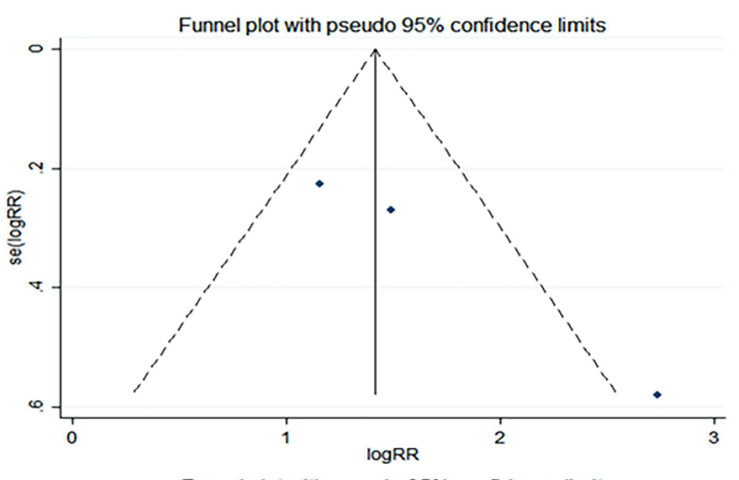

Funnel plot with pseudo $95 \%$ confidence limits

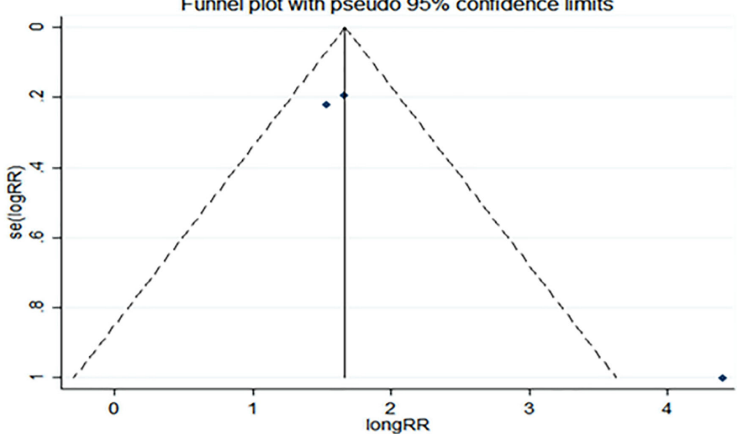

Fig. 4. Forest plots showing relative risks (RRs) between survivors and non-survivors of COVID-19. Each square shows effect estimate of individual studies with their $95 \%$ CI. Size of squares is proportional to the weight of each study in the meta-analysis. In this plot, studies are shown in the order of publication date and first author's names. Funnel plot for publication bias. 


\section{Limitations}

This study had several limitations. First, in our included literature, there is no clear definition myocardial injury and arrhythmia in COVID-19 patients. Second, all of most of the studies were from Wuhan, China and the possibility of overlap was high, so the same patients were likely to be included in multiple studies. Third, due to the small sample size and limited time, the data collection was incomplete and most of the studies did not analyze complications in non-survivors. Hence, we could not conduct sensitivity analysis and subgroup analysis. Larger studies are needed to confirm our findings. Finally, high statistical heterogeneity was found. All included studies were retrospective and there was risk of bias in the collected data.

\section{Conclusions}

Myocardial injury and arrhythmia were the most common complications in COVID-19 patients. Myocardial injury and heart failure were more common in non-survivors, with or without a history of cardiovascular disease. The incidence of heart failure and myocardial injury were higher in non-survivors compared to the survivors. Future studies on COVID-19 need to specifically describe the mechanisms and outcomes of various cardiovascular disease manifestations, as well as the cardiovascular effects during pharmacotherapy.

\section{Author contributions}

ZYH and ZL analyzed the data and wrote the manuscript. YXC and WP contributed to the final version of the manuscript.

\section{Acknowledgment}

I would like to express my gratitude to all those who helped me during the writing this manuscript.

\section{Funding}

This research received no external funding.

\section{Conflict of interest}

The authors claim no conflict of interest.

\section{References}

[1] WHO. WHO Coronavirus Disease (COVID-19) Dashboard. 2020.

[2] Madjid M, Safavi-Naeini P, Solomon SD, Vardeny O. Potential effects of coronaviruses on the cardiovascular system. JAMA Cardiology. 2020; 5: 831.

[3] Aggarwal G, Cheruiyot I, Aggarwal S, Wong J, Lippi G, Lavie CJ, et al. Association of cardiovascular disease with coronavirus disease 2019 (COVID-19) severity: a meta-analysis. Current Problems in Cardiology. 2020; 45: 100617.

[4] Huang C, Wang Y, Li X, Ren L, Zhao J, Hu Y, et al. Clinical features of patients infected with 2019 novel coronavirus in Wuhan, China. The Lancet. 2020; 395: 497-506.

[5] Hoffmann M, Kleine-Weber H, Schroeder S, Krüger N, Herrler $\mathrm{T}$, Erichsen S, et al. SARS-CoV-2 cell entry depends on ACE2 and
TMPRSS2 and is blocked by a clinically proven protease inhibitor. Cell. 2020; 181: 271-280.e8.

[6] Oudit GY, Kassiri Z, Jiang C, Liu PP, Poutanen SM, Penninger JM, et al. SARS-coronavirus modulation of myocardial ACE2 expression and inflammation in patients with SARS. European Journal of Clinical Investigation. 2009a; 39: 618-625.

[7] Xu Z, Shi L, Wang Y, Zhang J, Huang L, Zhang C, et al. Pathological findings of COVID-19 associated with acute respiratory distress syndrome. The Lancet Respiratory Medicine. 2020; 8: 420422.

[8] Argenziano MG, Bruce SL, Slater CL, Tiao JR, Baldwin MR, Barr RG, et al. Characterization and clinical course of 1000 patients with coronavirus disease 2019 in New York: retrospective case series. British Medical Journal. 2020; 369: m1996.

[9] Buckner FS, McCulloch DJ, Atluri V, Blain M, McGuffin SA, Nalla $\mathrm{AK}$, et al. Clinical features and outcomes of 105 hospitalized patients with COVID-19 in Seattle, Washington. Clinical Infectious Diseases. 2020; 71: 2167-2173.

[10] Chen T, Wu D, Chen H, Yan W, Yang D, Chen G, et al. Clinical characteristics of 113 deceased patients with coronavirus disease 2019: retrospective study. British Medical Journal. 2020; 368: m1091.

[11] Du Y, Tu L, Zhu P, Mu M, Wang R, Yang P, et al. Clinical features of 85 fatal cases of COVID-19 from Wuhan. A retrospective observational study. American Journal of Respiratory and Critical Care Medicine. 2020; 201: 1372-1379.

[12] Guo T, Fan Y, Chen M, Wu X, Zhang L, He T, et al. Cardiovascular implications of fatal outcomes of patients with coronavirus disease 2019 (COVID-19). JAMA Cardiology. 2020; 5: 811.

[13] Lodigiani C, Iapichino G, Carenzo L, Cecconi M, Ferrazzi P, Sebastian $\mathrm{T}$, et al. Venous and arterial thromboembolic complications in COVID-19 patients admitted to an academic hospital in Milan, Italy. Thrombosis Research. 2020; 191: 9-14.

[14] Shi M, Chen L, Yang Y, Zhang J, Xu J, Xu G, et al. Analysis of clinical features and outcomes of 161 patients with severe and critical COVID-19: a multicenter descriptive study. Journal of Clinical Laboratory Analysis. 2020; 34: e23415.

[15] Wan S, Xiang Y, Fang W, Zheng Y, Li B, Hu Y, et al. Clinical features and treatment of COVID-19 patients in northeast Chongqing. Journal of Medical Virology. 2020; 92: 797-806.

[16] Wang D, Hu B, Hu C, Zhu F, Liu X, Zhang J, et al. Clinical characteristics of 138 hospitalized patients with 2019 novel coronavirusinfected pneumonia in Wuhan, China. The Journal of the American Medical Association. 2020; 323: 1061.

[17] Zhou F, Yu T, Du R, Fan G, Liu Y, Liu Z, et al. Clinical course and risk factors for mortality of adult inpatients with COVID-19 in Wuhan, China: a retrospective cohort study. The Lancet. 2020; 395: 1054-1062.

[18] Wang L, He W, Yu X, Hu D, Bao M, Liu H, et al. Coronavirus disease 2019 in elderly patients: characteristics and prognostic factors based on 4-week follow-up. Journal of Infection. 2020; 80: 639645.

[19] Nguyen JL, Yang W, Ito K, Matte TD, Shaman J, Kinney PL Seasonal influenza infections and cardiovascular disease mortality. JAMA Cardiology. 2016; 1: 274.

[20] Cameron MJ, Ran L, Xu L, Danesh A, Bermejo-Martin JF, Cameron CM, et al. Interferon-mediated immunopathological events are associated with atypical innate and adaptive immune responses in patients with severe acute respiratory syndrome. Journal of Virology. 2007; 81: 8692-8706.

[21] Babapoor-Farrokhran S, Rasekhi RT, Gill D, Babapoor S, Amanullah A. Arrhythmia in COVID-19. SN Comprehensive Clinical Medicine. 2020; 2: 1430-1435.

[22] Zheng Y, Ma Y, Zhang J, Xie X. COVID-19 and the cardiovascular system. Nature Reviews Cardiology. 2020; 17: 259-260. 
[23] Driggin E, Madhavan MV, Bikdeli B, Chuich T, Laracy J, BiondiZoccai G, et al. Cardiovascular considerations for patients, health care workers, and health systems during the COVID-19 pandemic. Journal of the American College of Cardiology. 2020; 75: 23522371.

[24] Clayton TC, Thompson M, Meade TW. Recent respiratory infection and risk of cardiovascular disease: case-control study through a general practice database. European Heart Journal. 2008; 29: 96103.

[25] Hamadeh A, Aldujeli A, Briedis K, Tecson KM, Sanz-Sánchez J, Al dujeili $\mathrm{M}$, et al. Characteristics and outcomes in patients presenting with COVID-19 and ST-segment elevation myocardial infarction. American Journal of Cardiology. 2020; 131: 1-6.

[26] Elsaid O, McCullough PA, Tecson KM, Williams RS, Yoon A. Ventricular fibrillation storm in coronavirus 2019. American Journal of Cardiology. 2020; 135: 177-180.

[27] Corrales-Medina VF, Madjid M, Musher DM. Role of acute infection in triggering acute coronary syndromes. The Lancet Infectious Diseases. 2010; 10: 83-92.

[28] Chen N, Zhou M, Dong X, Qu J, Gong F, Han Y, et al. Epidemiological and clinical characteristics of 99 cases of 2019 novel coro- navirus pneumonia in Wuhan, China: a descriptive study. The Lancet. 2020; 395: 507-513.

[29] Bansal M. Cardiovascular disease and COVID-19. Diabetes \& Metabolic Syndrome: Clinical Research \& Reviews. 2020; 14: 247250.

[30] Limsreng S, Marcy O, Ly S, Ouk V, Chanroeurn H, Thavary S, et al. Dyslipidemias and elevated cardiovascular risk on lopinavirbased antiretroviral therapy in Cambodia. PLoS ONE. 2016; 11: e0160306.

[31] Trac MH, McArthur E, Jandoc R, Dixon SN, Nash DM, Hackam DG, et al. Macrolide antibiotics and the risk of ventricular arrhythmia in older adults. Canadian Medical Association Journal. 2016; 188: E120-E129.

[32] Chorin E, Wadhwani L, Magnani S, Dai M, Shulman E, NadeauRouthier C, et al. QT interval prolongation and torsade de pointes in patients with COVID-19 treated with hydroxychloroquine/azithromycin. Heart Rhythm. 2020; 17: 1425-1433.

[33] Briedis K, Aldujeli A, Aldujeili M, Briede K, Zaliunas R, Hamadeh $A$, et al. Considerations for management of acute coronary syndromes during the SARS-CoV-2 (COVID-19) pandemic. American Journal of Cardiology. 2020; 131: 115-119. 